Journal of Patient-Centered

Volume 7

Issue 4 -- Women and Cancer

Article 4

$10-23-2020$

\title{
Evaluation of a Mobile Health Intervention to Improve Wellness Outcomes for Breast Cancer Survivors
}

Jamie Cairo

Laurie Williams

Lisa Bray

Katrina Goetzke

Ana Cristina Perez

Follow this and additional works at: https://aah.org/jpcrr

Part of the Dietetics and Clinical Nutrition Commons, Health Psychology Commons, Neoplasms Commons, Oncology Commons, and the Women's Health Commons

\section{Recommended Citation}

Cairo J, Williams L, Bray L, Goetzke K, Perez AC. Evaluation of a mobile health intervention to improve wellness outcomes for breast cancer survivors. J Patient Cent Res Rev. 2020;7:313-22. doi: 10.17294/ 2330-0698.1733

Published quarterly by Midwest-based health system Advocate Aurora Health and indexed in PubMed Central, the Journal of Patient-Centered Research and Reviews (JPCRR) is an open access, peer-reviewed medical journal focused on disseminating scholarly works devoted to improving patient-centered care practices, health outcomes, and the patient experience. 


\title{
Evaluation of a Mobile Health Intervention to Improve Wellness Outcomes for Breast Cancer Survivors
}

\author{
Jamie Cairo, DNP, AOCNP, ${ }^{1}$ Laurie Williams, MSN, APNP, ${ }^{1}$ Lisa Bray, FNP, AOCNP, ${ }^{1}$ Katrina Goetzke, \\ $\mathrm{PA}-\mathrm{C},{ }^{1}$ Ana Cristina Perez, MD, $\mathrm{PhD}^{2}$ \\ ${ }^{1}$ Aurora Cancer Care, Advocate Aurora Health, Milwaukee, WI; ${ }^{2}$ Advocate Aurora Research Institute, Advocate Aurora \\ Health, Milwaukee, WI
}

Purpose Breast cancer survivors are at increased risk of cancer recurrence, second malignancies, and other comorbid conditions. This study examined if use of a convenient, commercially available, $\$ 65$ per month app that gives breast cancer survivors access to a health and wellness coach is more effective than a self-guided toolkit and one-time health education session at achieving the following goals: 1) improving adherence to a plant-based diet, 2) increasing physical activity, 3) assisting with weight loss and reduction in body mass index, 4) reducing elevated depression and fatigue scores, and 5) leading to sustained adherence to lifestyle and wellness plan at and beyond 6 months.

Methods A nonrandomized 2-group control study design with pre-post repeated measures ( $N=127$ subjects) was utilized. Women 18 years of age or older, with curative-intent breast cancer, were included in the study. App users received a survivorship care plan and enrolled in a 6-month subscription to the health app. A control group received the same information but, instead of access to the app, were given a self-guided toolkit.

Results At 6 months, more patients in the app group experienced weight loss and had a significantly greater reduction in overall body mass index $(P<0.01)$. The app group also demonstrated statistically significant improvements in "strenuous" physical activity $(P=0.04)$ and had significant improvement in their dietary patterns $(P<0.001)$, as compared to the self-guided group. The app group had greater reduction in fatigue and improvement in depression, but these changes were not statistically significant. At 12 months, none of the app users were still using the app, but many were still following their wellness plan and had maintained their weight loss. Outliers in both groups and low rate of response made evaluation of results difficult.

Conclusions The results of this advanced practice provider-led study demonstrated that a live health coaching app that provides wellness coaching can offer motivated breast cancer survivors and cancer programs a modality that offers convenient, effective support at a reasonable cost. ( $J$ Patient Cent Res Rev. 2020;7:313-322.)

Keywords mobile health; wellness coaching; breast cancer; survivorship; nutrition; physical activity

$\mathrm{I}$ $\mathrm{n}$ the United States, 1 in 8 women will develop breast cancer over the course of a lifetime. The current average 10 -year survival rate is $83 \%$; if the cancer has not spread outside of the breast, the 5-year survival rate is $99 \%{ }^{1,2}$ The increased number of breast cancer survivors can be attributed to both better screening and treatment.

Corresponding author: Jamie Cairo, DNP, AOCNP, Aurora Cancer Care, 3000 W. Montana Street, Milwaukee, WI 53215 (jamie.cairo@aah.org)
However, with more survivors, there are now more reported long-term physical and psychological issues that can lead to chronic latent side effects and symptoms. ${ }^{3}$ Breast cancer survivors are at increased risk of cancer recurrence, secondary malignancies, and other comorbid conditions including diabetes, osteoporosis, obesity, cardiovascular disease, and psychosocial distress. ${ }^{3-6}$

In the general cancer survivor population, a plant-based diet that is rich in fruits, vegetables, and whole grains has been linked to prolonged survival in populationbased studies. ${ }^{7,8}$ For patients with breast cancer, dietary 
modification may be particularly important. Patients with certain breast tumor types may benefit more directly than others from dietary intervention. For instance, patients with estrogen- and/or progesteronepositive tumors have been found to have reduced recurrence rates when they follow a low-fat plant-based diet. ${ }^{9}$ Additionally, there is a direct link to obesity in the general breast cancer survivor population, and obesity is known to be a risk factor for recurrence in breast cancer survivors. ${ }^{10,11}$ A 2011 review found that $50 \%$ to $96 \%$ of women with breast cancer reported weight gain during treatment, and those who did not gain weight during treatment often gained over the next 3 years following treatment. ${ }^{12}$ In fact, Nichols et al reported that each 5-lb weight gain postdiagnosis was associated with a $13 \%$ increase in breast cancer-specific mortality and a $12 \%$ increase in all-cause mortality. ${ }^{13}$

Exercise has been shown to improve bone health, cardiovascular wellness, muscle and fat composition, chronic pain, insomnia, fatigue, anxiety, depression, selfesteem, and other components of quality of life in cancer survivors. ${ }^{14-16} \mathrm{~A}$ review by Spei et al of 10 observational studies found that cancer survivors who were more physically active have a lower risk of cancer recurrence and improved survival compared with those who are inactive. ${ }^{17}$ Thus, achieving and maintaining a healthy weight, consuming a nutrient-rich, plant-based diet, and engaging in regular physical activity are all important factors in improving the health and overall well-being of breast cancer survivors.

For these reasons, the American Cancer Society/ American Society of Clinical Oncology Breast Cancer Survivorship Care Guideline recommends that survivors maintain an optimum weight and engage in healthy lifestyle habits to reduce risk of recurrence, mortality, and other chronic diseases. ${ }^{7}$ After a cancer diagnosis and subsequent treatment, survivors are often highly motivated to seek information about positive lifestyle choices such as healthy eating and increased physical activity that can improve their treatment outcomes, quality of life, and overall survival. Despite this motivation and knowledge of the benefits, many survivors do not pursue or struggle to sustain healthy lifestyle habits. ${ }^{18-21}$

In terms of exercise and overall fitness, adherence can be defined as the degree of attendance or completion of prescribed exercise sessions either in a supervised setting or on one's own. ${ }^{22}$ The reasons for lack of adherence are many and varied. Variables predicting higher adherence to an exercise intervention after cancer treatment span sociodemographic, physical, physiological, and behavioral factors. Survivors who have higher levels of self-efficacy and motivation, family support, and the availability of feedback by coaches and trainers are more likely to adhere to an intervention..$^{23}$

There is an emerging consensus that health and wellness coaching, a patient-centered process that is based on behavior change theory, may help patients achieve their lifestyle and fitness goals. ${ }^{24}$ Wellness coaches are trained certified specialists who help clients optimize their health and wellness by building a system of self-efficacy, creating realistic individualized lifestyle changes and then providing ongoing support and reinforcement of behaviors using motivational techniques. Wellness coaches are typically nurses, dieticians, fitness trainers, or therapists who have a background and experience with different health conditions. Barakat et al conducted a systematic review involving 12 studies of health coaching interventions with 1038 all-cause cancer survivors. ${ }^{25}$ They found that health coaching improved quality of life, lessened chronic pain and fatigue, and increased physical activity.

Despite the benefits of wellness coaching, the cost of individual coaching can be prohibitive, ranging from $\$ 50-\$ 300$ a session (in southeastern Wisconsin), and it can be difficult to find a coach depending on location. There are several self-guided resources available to patients, including websites and commercially available mobile apps. One such product named Vida (Vida Health) is a mobile health coaching app with a unique platform that focuses on improving health and wellness outcomes by pairing users with a trained wellness coach who connects with them via smartphone, email, or phone daily for a subscription fee of $\$ 65$ per month. The goal is to provide affordable access to a personalized wellness plan, real-time health support, and advice as it relates to weight loss, improved nutrition, fitness, stress reduction, or management of other chronic health conditions. A small pilot study conducted by researchers at Duke Health (Durham, NC) demonstrated that the Vida mobile health platform approach can help cardiac rehab patients stay on track with their wellness goals after supervised cardiac rehab had ended and also appeared to assist users in continuing to increase aerobic endurance. ${ }^{26}$ Another retrospective study with over 1000 participants found that Vida app users lost an average of $3.23 \%$ total body weight at 4 months of coaching, and $28.6 \%$ achieved a clinically significant weight loss of $5 \%$ or more of total body weight. ${ }^{27}$

The purpose of this study was to evaluate if a readily available mobile health intervention, ie, Vida, can lead to healthier lifestyle habits for breast cancer survivors. 


\section{METHODS}

\section{Study Design}

The study was approved by the institutional review board of Aurora Health Care (Milwaukee, WI). Women 18 years of age or older with curative-intent (stage $0-$ III) breast cancer who were not currently enrolled in any other wellness studies were eligible for participation. Patients with metastatic breast cancer were excluded because their survivorship needs differ from curative-intent patients.

A pragmatic, nonrandomized 2-group control design with pre-post repeated measures was utilized for this investigation. After the study was explained to potential participants, an assessment was done to see if they had personal access to a smartphone and the ability and willingness to download and use the Vida app (Vida group). Those who did not have access or the willingness to interact with an app were assigned to the self-guided toolkit (Self group). Both groups received a printed survivorship care plan that included evidence-based nutrition and activity recommendations from an advanced practice provider.

Participants in the intervention arm were enrolled in a 6-month subscription to the Vida app, through which they were paired with a certified wellness coach who oriented them to use of the app. Participants were matched with a coach based on their biometrics, health goals, and desired coaching style. The coaches maintained regular video/ phone consultation and provided daily motivation through messaging. Patients were able to track their medication, diet, exercise, sleep, and weight through the app, and their coach could give feedback based on that data.

The Self group (control arm) received the same printed survivorship information, but instead of the Vida intervention they were given a self-guided "toolkit" that included additional nutrition information, an exercise stretch band, a pedometer, and a self-guided walking DVD.

Both groups answered the same questionnaires at baseline and again at 6 months. Weight and body mass index (BMI) also were measured at baseline and at 6 months.

\section{Instruments}

Four tools were used to measure the variables of interest. The 4-item Godin-Shephard Leisure-Time Physical Activity Questionnaire is used to assess the number of times one engages in mild, moderate, and strenuous physical activity of at least 15 minutes duration in a typical week. ${ }^{28}$ The "Rate Your Plate" nutritional assessment is a 27 -item survey that assigns a score based on response, with higher scores indicating better adherence to a balanced and healthy diet. ${ }^{29}$ The
Visual Analog Scale - Fatigue is a validated singleitem measure designed specifically for use with cancer patients and was used to assess the presence and severity of fatigue. ${ }^{30}$ The Patient Health Questionnaire (PHQ-2) depression tool asks about the frequency of depressed mood and anhedonia over the previous 2 weeks. ${ }^{31}$

At 12 months, participants were contacted, primarily by email, and asked if they were still using the app or toolkit, to reflect on what they liked and disliked about the app or toolkit, their current weight, and if they were still following their individual wellness plan.

\section{Statistical Analysis}

Baseline characteristics are summarized as counts and percentages for categorical variables and means and standard deviations (SD) for continuous variables. Baseline demographic and response characteristics were compared between the 2 groups by standard descriptive statistic methods ( $t$-test and Pearson's chi-squared). Follow-up characteristics at 6 months were compared in the same manner. Before and after responses within each group were compared by paired $t$-test for continuous variables.

Responses also were classified as no change, improved, or worsening, and categories were compared between the 2 groups. Given that the groups are independent, these categorical response variables (presented as counts and proportions in figures) were compared between the 2 groups using a chi-squared test. A P-value of $<0.05$ was considered statistically significant. All statistical analyses were done using Stata 15 software (StataCorp LLC).

\section{RESULTS}

Baseline demographic data (Table 1) found that both arms of the study were predominantly White and not of Hispanic/Latino ethnicity. The mean age for the Self (toolkit) group was 56.7 years, while mean age for the Vida group was 51.4 years. Baseline BMIs were similar. Most of the study participants in both groups had been diagnosed with early-stage breast cancer, but a higher percentage of the Vida group had later (stage III) cancer diagnoses. In all, 61 subjects in the Self group completed their weights and surveys, and 66 in the Vida group completed the full 6-month intervention as well as weights and surveys.

At the end of the 6-month intervention period, 16 participants chose to drop out of the study, with the most common reasons given that they changed their minds after signing consent or found that the app was "annoying" or too much work for them. Four participants were dropped from the study at the 6-month 
Table 1. Demographics and Baseline Data of Study Groups

\begin{tabular}{|c|c|c|c|}
\hline Variable & $\begin{array}{c}\text { Self } \\
n=61\end{array}$ & $\begin{array}{l}\text { Vida } \\
n=66\end{array}$ & $P^{c}$ \\
\hline Age in years, mean (SD) & $56.7(9.8)$ & $51.4(8.1)$ & 0.001 \\
\hline Stage of breast cancer, $\mathrm{n}(\%)$ & & & 0.81 \\
\hline 0 & $6(10 \%)$ & $6(9 \%)$ & \\
\hline 1 & $1(2 \%)$ & $3(5 \%)$ & \\
\hline la & $23(38 \%)$ & $24(36 \%)$ & \\
\hline $\mathrm{lb}$ & $1(2 \%)$ & $2(3 \%)$ & \\
\hline Ila & $17(28 \%)$ & $11(17 \%)$ & \\
\hline Illb & $5(8 \%)$ & $8(12 \%)$ & \\
\hline IIla & $4(7 \%)$ & $3(5 \%)$ & \\
\hline IIlb & $1(2 \%)$ & $2(3 \%)$ & \\
\hline IIIC & $1(2 \%)$ & $3(5 \%)$ & \\
\hline Unknown & $2(3 \%)$ & $4(6 \%)$ & \\
\hline Preintervention weight in kg, mean (SD) & $84.2(21.5)$ & $81.5(19.5)$ & 0.46 \\
\hline Preintervention BMI, mean (SD) & $30.2(7.3)$ & $29.4(6.0)$ & 0.47 \\
\hline Ethnicity, n (\%) & & & 0.96 \\
\hline Hispanic/Latino & $1(2 \%)$ & $1(2 \%)$ & \\
\hline Not Hispanic/Latino & $60(98 \%)$ & $65(98 \%)$ & \\
\hline Race, n (\%) & & & 0.63 \\
\hline Asian & $0(0 \%)$ & $1(2 \%)$ & \\
\hline Black/African American & $3(5 \%)$ & $3(5 \%)$ & \\
\hline White & $58(95 \%)$ & $62(94 \%)$ & \\
\hline Baseline Godin-Shephard score, mean (SD) & $5.7(4.3)$ & $6.4(4.1)$ & 0.35 \\
\hline Baseline Godin-Shephard activity level, n (\%) & & & 0.78 \\
\hline Insufficient & $58(95 \%)$ & $62(94 \%)$ & \\
\hline Moderate & $3(5 \%)$ & $4(6 \%)$ & \\
\hline Baseline Rate Your Plate score, mean (SD) & $53.2(6.9)$ & $52.7(7.2)$ & 0.70 \\
\hline Baseline fatigue score, mean (SD) & $4.3(2.0)$ & $4.8(2.4)$ & 0.22 \\
\hline Baseline depression, ${ }^{a}$ n (\%) & $19(31 \%)$ & $21(32 \%)$ & 0.94 \\
\hline Baseline pleasure, ${ }^{\mathrm{b}} \mathrm{n}(\%)$ & $19(31 \%)$ & $15(23 \%)$ & 0.28 \\
\hline
\end{tabular}

a During the past month, have you often been bothered by feeling down, depressed?

${ }^{b}$ During the past month, have you often been bothered by little interest or pleasure?

${ }^{c}$ Determined by t-test and Pearson's chi-squared.

$B M I$, body mass index; $S D$, standard deviation.

mark because they declined to answer the follow-up questions. One study subject passed away (diagnosis not related to cancer) during the study, and 2 others were diagnosed with metastatic disease and did not complete the study.

An overall comparison summary between the 2 groups found that the most statistically significant difference preand postintervention was adherence to a plant-based diet as measured by the Rate Your Plate tool (Table 2).

\section{Weight Gain/Loss}

More patients in the Vida group experienced weight loss compared to the Self group and had a greater reduction in overall BMI (Figure 1). The average for the Vida group was a $1.8-\mathrm{kg}$ loss (mean $\Delta: 1.8, \mathrm{SD}: 4.9,95 \% \mathrm{CI}: 0.6$ to $3.0 ; \mathrm{P}<0.01$ ) and reduction in BMI (mean $\Delta: 0.7$, SD: $1.8,95 \%$ CI: 0.2 to $1.1 ; \mathrm{P}<0.01$ ) that was statistically significant compared to the Self group, who on average had a weight gain of $0.14 \mathrm{~kg}$ (mean $\Delta:-0.2, \mathrm{SD}: 3.7,95 \%$ CI: -1.1 to $0.8 ; \mathrm{P}=0.70$ ) and $\mathrm{BMI}$ changes that were not 
Table 2. Results at 6-Month Follow-Up

\begin{tabular}{|c|c|c|c|}
\hline Outcome measure & $\begin{array}{c}\text { Self } \\
n=61\end{array}$ & $\begin{array}{l}\text { Vida } \\
n=66\end{array}$ & $P^{c}$ \\
\hline Follow-up weight in kg, mean (SD) & $84.4(21.7)$ & $79.7(19.2)$ & 0.20 \\
\hline Postintervention BMI, mean (SD) & $30.3(7.4)$ & $28.7(5.9)$ & 0.18 \\
\hline Follow-up Godin-Shephard score, mean (SD) & $7.7(5.9)$ & $8.2(5.2)$ & 0.64 \\
\hline Follow-up Godin-Shephard activity level, n (\%) & & & 0.71 \\
\hline Active & $2(3 \%)$ & $1(2 \%)$ & \\
\hline Moderate & $8(13 \%)$ & $11(17 \%)$ & \\
\hline Insufficient & $51(84 \%)$ & $54(82 \%)$ & \\
\hline Follow-up Rate Your Plate score, mean (SD) & $55.2(6.7)$ & $58.9(6.2)$ & $<0.001$ \\
\hline Follow-up fatigue score, mean (SD) & $3.7(3.4)$ & $3.6(2.3)$ & 0.82 \\
\hline Follow-up depression, ${ }^{a}$ n (\%) & & & 0.43 \\
\hline Unknown & $1(2 \%)$ & $0(0 \%)$ & \\
\hline Yes & $15(25 \%)$ & $13(20 \%)$ & \\
\hline Follow-up pleasure, ${ }^{\mathrm{b}} \mathrm{n}(\%)$ & & & 0.25 \\
\hline Unknown & $1(2 \%)$ & $0(0 \%)$ & \\
\hline Yes & $11(18 \%)$ & $7(11 \%)$ & \\
\hline
\end{tabular}

aDuring the past month, have you often been bothered by feeling down, depressed?

${ }^{b}$ During the past month, have you often been bothered by little interest or pleasure?

'Determined by t-test and Pearson's chi-squared.

$B M I$, body mass index; $S D$, standard deviation.

statistically significant (mean $\Delta:-0.7$, SD: $1.4,95 \% \mathrm{CI}$ : -0.4 to $0.3 ; \mathrm{P}=0.68)$. Overall, 40 of 66 subjects $(61 \%)$ in the Vida group lost weight and reduced BMI compared with 22 of 61 subjects $(36 \%)$ in the Self group.

\section{Physical Activity per Godin-Shephard}

Both groups had similar levels of activity at baseline and overall had similar levels of activity postintervention (Table 3). However, the Vida group demonstrated improvements in "strenuous activity" $(57.6 \%$ at baseline and $68.2 \%$ postintervention) that were statistically significant $(\mathrm{P}=0.04)$.

\section{Rate Your Plate Nutrition Assessment}

Adherence to a balanced diet that emphasizes plant-based foods was assessed using the Rate Your Plate assessment at baseline and at the end of the intervention (6 months). Postintervention scores showed that the Vida group had a highly statistically significant improvement in their dietary patterns (mean $\Delta$ : -6.2 , SD: $5.8,95 \% \mathrm{CI}:-7.6$ to -4.8 ; $\mathrm{P}<0.001$ ), with $80 \%$ of participants on that arm of the study reporting improved dietary intake (Figure 2) versus $51 \%$ of those in the Self group (mean $\Delta:-2.0, \mathrm{SD}$ : $6.5,95 \% \mathrm{CI}:-3.7$ to -0.3 ; P 0.02 ).

\section{Fatigue}

Both groups had a decrease in fatigue (Self group mean $\Delta$ : 0.65 , SD: $2.3,95 \%$ CI: 0.8 to 1.23 ; $\mathrm{P}=0.03$ ), but the Vida group had a greater reduction in symptoms (mean $\Delta: 1.2$, SD: $2.4,95 \%$ CI: 0.7 to $1.8 ; \mathrm{P}<0.001$ ), with $62 \%$ experiencing improvement compared to $49 \%$ in the Self group, a statistically significant difference. The Vida group also had fewer subjects reporting a worsening of their fatigue (20\%) in comparison to the $30 \%$ reported by the Self group (Figure 3).

\section{Depression}

The results were mixed between the 2 groups. In the Vida group, more participants who had reported depression at baseline (21\%) versus those in the Self group (12\%) experienced an improvement in their depression at the end of the intervention (Figure 4). However, the change was not statistically significant $(\mathrm{P}=0.359)$. 


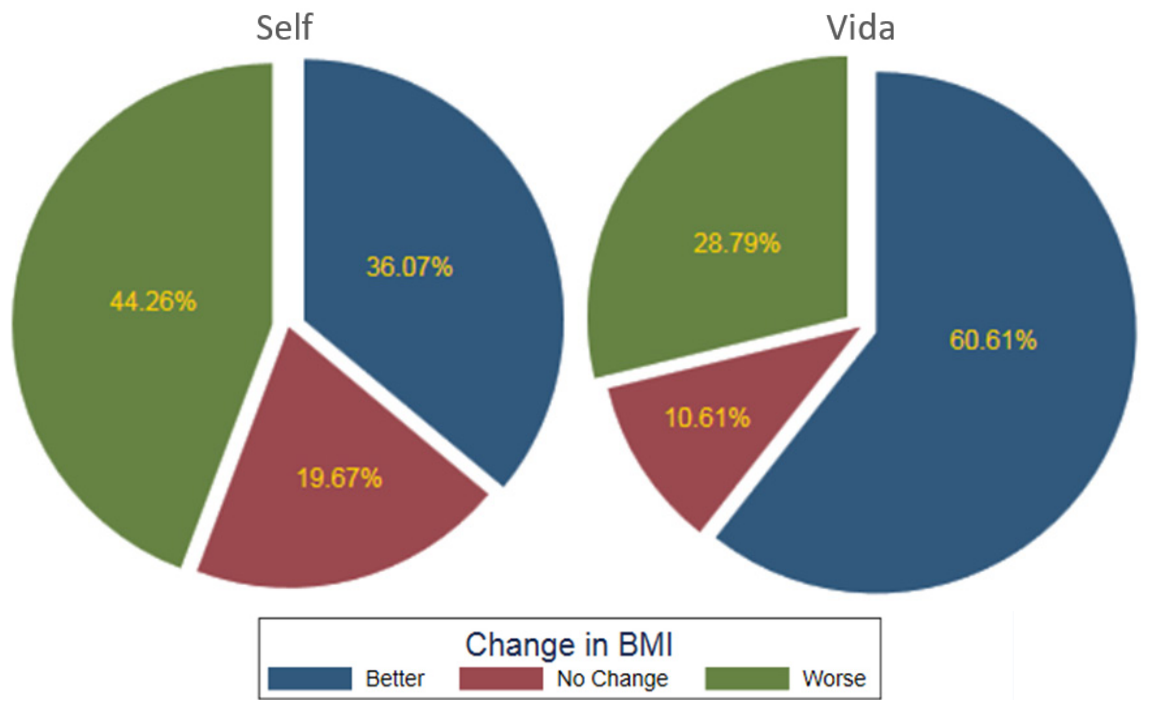

Figure 1. Postintervention body mass index changes between the Self and Vida groups.

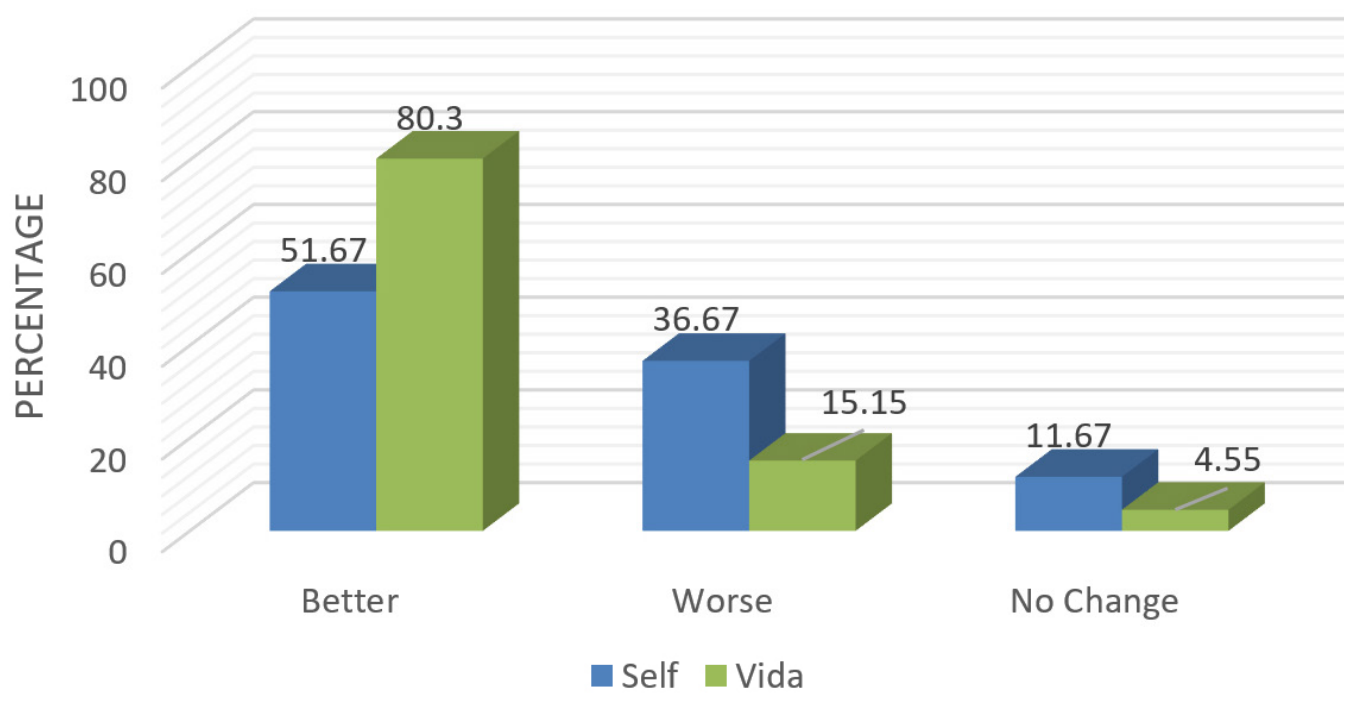

Figure 2.

Postintervention Rate Your Plate scores between the Self and Vida groups.

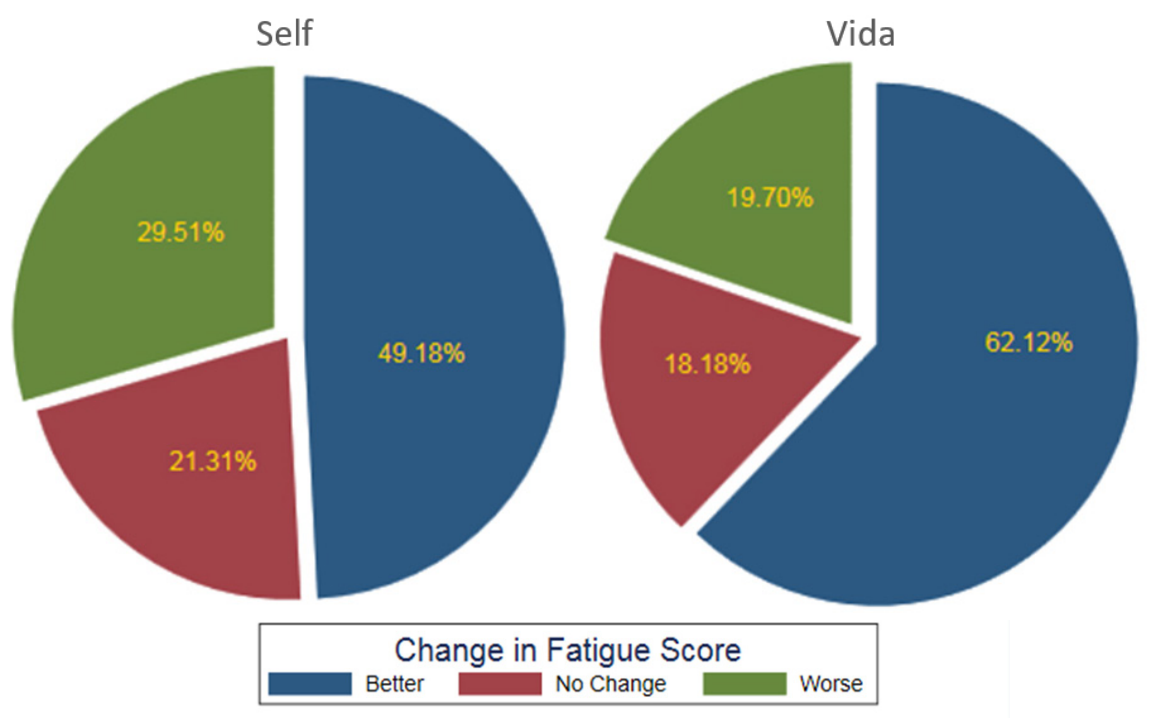

Figure 3. Postintervention change in fatigue between the Self and Vida groups. 

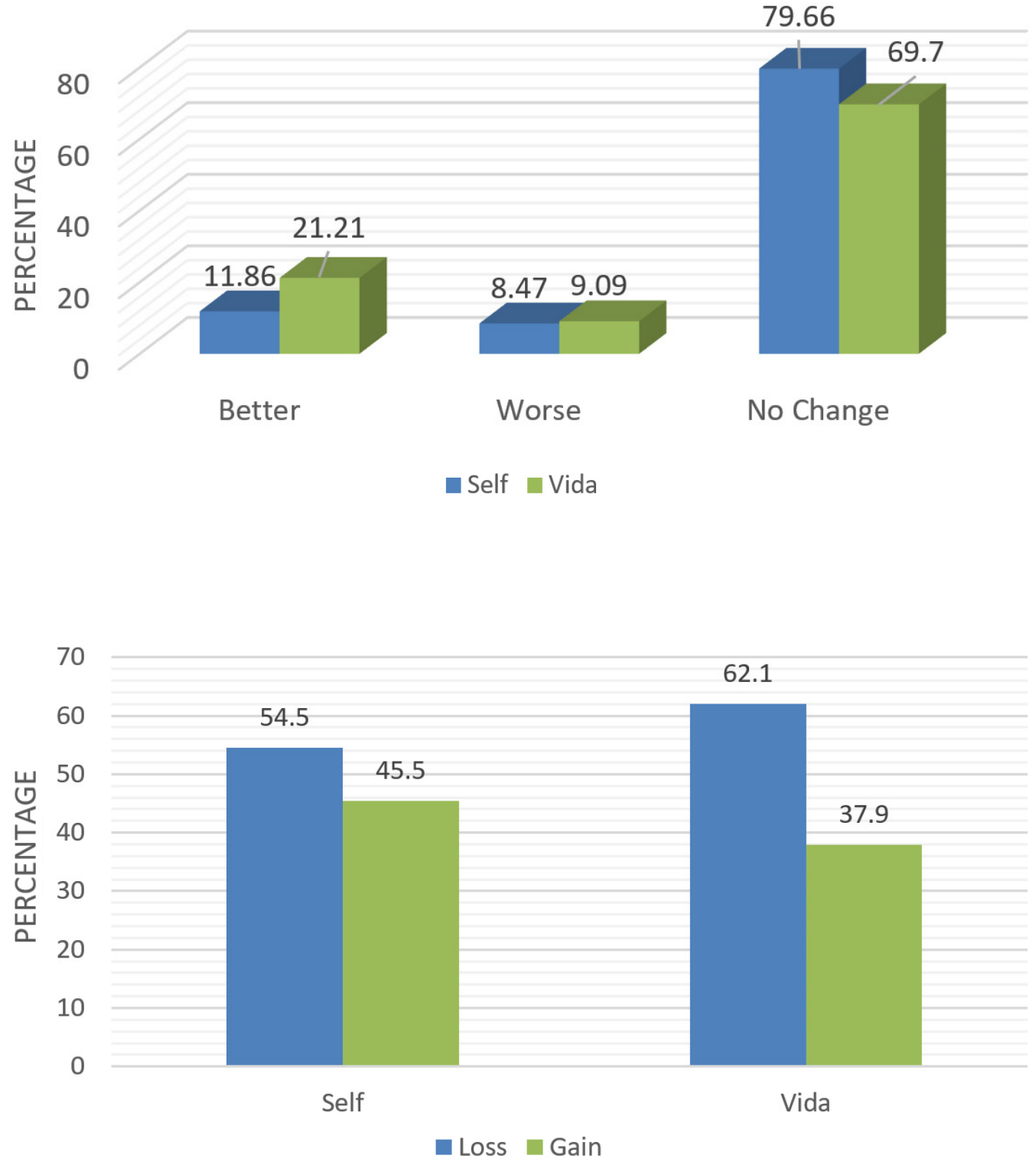

Figure 4.

Postintervention changes in Patient Health Questionnaire-2 depression scores between the Self and Vida groups.
Figure 5. Postintervention self-reported weight changes at 1 year.

\section{2-Month Follow-Up}

From the 127 participants who completed the 6-month intervention, 41 (30 Vida, 11 Self) replied to the 12-month questionnaire, providing an overall response rate of $32 \%$ ( $45 \%$ for the Vida group). None of the respondents were still using the Vida app, with most citing cost as the primary factor. For those who had participated in the Vida group, only 1 said they did not like the experience. The overwhelming majority felt that their interactions with the wellness coach was instrumental to their success. Sample quotes included:

- "Having a realperson to bounce ideas off of, ask healthrelated questions, and get immediate feedback."

- "My discussions with the coach kept me accountable, kept me focused."
- "I liked taking pictures of the food and sending to coach. Fast and easy."

- "I trusted my coach. She was patient, knowledgeable, upbeat, and supportive."

The negative feedback for the app users was mostly centered on technical difficulties in using the app (primarily syncing issues with exercise trackers and devices), scheduling, and time management related to effective use of the app. The mean difference obtained by $t$-test in self-reported weights preintervention and 12-month follow-up between the Self and Vida groups was $0.72 \mathrm{~kg}(95 \% \mathrm{CI}$ : -0.89 to 2.27; $\mathrm{P}=0.89$ ). Patients enrolled in the Vida group lost an average of $0.79 \mathrm{~kg}$ while those enrolled in the Self group lost an average of $0.55 \mathrm{~kg}$ (Figure 5). These differences, however, were not statistically significant. 
Table 3. Baseline and Postintervention Activity Levels Between the Self and Vida Groups

\begin{tabular}{|c|c|c|c|}
\hline Godin-Shephard variable & $\begin{array}{c}\text { Self } \\
n=61\end{array}$ & $\begin{array}{l}\text { Vida } \\
\mathrm{n}=66\end{array}$ & $P^{a}$ \\
\hline Strenuous exercise at baseline, mean score (SD) & $5.0(11.3)$ & $9.0(13.2)$ & 0.07 \\
\hline Moderate exercise at baseline, mean score (SD) & $11.1(11.3)$ & $10.8(10.9)$ & 0.87 \\
\hline Mild exercise at baseline, mean score (SD) & $9.2(7.7)$ & $9.8(7.5)$ & 0.68 \\
\hline Total exercise at baseline, mean score (SD) & $25.4(19.6)$ & $29.6(20.6)$ & 0.24 \\
\hline Activity at baseline, $\mathrm{n}(\%)$ & & & 0.43 \\
\hline Active & $29(47.5 \%)$ & $38(57.6 \%)$ & \\
\hline Moderate & $14(23.0 \%)$ & $10(15.2 \%)$ & \\
\hline Insufficient & $18(29.5 \%)$ & $18(27.3 \%)$ & \\
\hline Strenuous exercise at follow-up, mean score (SD) & $6.9(13.5)$ & $12.2(15.6)$ & 0.04 \\
\hline Moderate exercise at follow-up, mean score (SD) & $15.0(16.0)$ & $14.9(11.8)$ & 0.98 \\
\hline Mild exercise at follow-up, mean score (SD) & $11.9(9.0)$ & $11.5(9.3)$ & 0.83 \\
\hline Total exercise at follow-up, mean score (SD) & $33.8(27.4)$ & $38.7(25.3)$ & 0.30 \\
\hline Activity at follow-up, n (\%) & & & 0.19 \\
\hline Active & $37(60.7 \%)$ & $45(68.2 \%)$ & \\
\hline Moderate & $8(13.1 \%)$ & $12(18.2 \%)$ & \\
\hline Insufficient & $16(26.2 \%)$ & $9(13.6 \%)$ & \\
\hline $\begin{array}{l}\text { Difference in total preactivity and postactivity scores, mean } \\
\text { increase (SD) }\end{array}$ & $8.4(29.1)$ & $9.1(24.6)$ & 0.89 \\
\hline Change in activity level, $\mathrm{n}(\%)$ & & & 0.54 \\
\hline No change & $3(4.9 \%)$ & $3(4.6 \%)$ & \\
\hline More active & $33(54.1 \%)$ & $42(63.6 \%)$ & \\
\hline Less active & $25(41.0 \%)$ & $21(31.8 \%)$ & \\
\hline
\end{tabular}

aProportions between the groups were compared using chi-squared test; continuous variables were compared using unpaired t-test.

$S D$, standard deviation.

Five participants used the app for a period after the 6-month intervention ended, but no participants continued to use the wellness coaching on their own at 12 months, even those who had found the app to be particularly helpful. Some of the app users said that they felt they had gained what they could from the app and no longer felt it was necessary.

The comparison of means at 12 months was affected by extreme values. For example, 1 patient on the Vida group gained $8.8 \mathrm{~kg}$ while another lost $12.1 \mathrm{~kg}$. On the other hand, one patient in the Self group gained $4.4 \mathrm{~kg}$ while the largest weight loss was $5.2 \mathrm{~kg}$. The more extreme values in the Vida group affected the mean in that group. It is also important to note that we do not know if any of these weight changes were in part due to muscle mass increase versus fat increase.

\section{DISCUSSION}

This study, which was designed and conducted by advanced practice providers in clinical practice at a large Midwestern cancer program, found statistically significant effects in the areas of nutrition, fatigue reduction, and increased time spent doing strenuous exercise by the Vida app users. The Vida group also lost more weight at 6 months than those in the Self (toolkit) group. It had been anticipated that some subjects would continue to use the app, on their own, after the 6-month trial period; while a few participants did, by 1 year none of the participants were still utilizing the app. Although cost was cited as the primary reason, it should be noted that the monthly cost of $\$ 65$ is significantly lower than the cost of individual face-to-face sessions with a health or wellness coach. 
This study was focused on breast cancer patients who may have distinct and different needs than other cancer survivors. Breast cancer survivors experience a number of long-term side effects related to their treatment and must also address preexisting comorbidities and lifestyle modifications to reduce risk of recurrence and improve overall health and well-being. ${ }^{32}$ We propose that this intervention is replicable in other patient populations and settings. Randomized studies with both breast cancer and other cancer survivors would be beneficial. It also would be useful to look at the effects of mobile health interventions over longer periods of time, which could allow for evaluation of improvements in morbidity and mortality. In future studies, it would be helpful to take additional measurements including waist size, skin fold testing, or other body fat analysis.

\section{Limitations}

There are some limitations to this study, including a relatively small sample size. The only variable that was statistically different at baseline was age; this is a limitation, as we did not adjust for age and participants were not matched for it. The study sampled only women with curative-intent breast cancer, and subjects were not randomized between the 2 arms because participants needed to have either smartphone or tablet access to participate in the Vida group. We cannot exclude the possibility of selection bias, as cancer survivors who chose to participate, particularly in the Vida group, may have been more motivated or more health-conscious than cancer survivors who declined to participate. Generally, participants did not respond to our follow-up phone calls at 12 months, so we adapted our communication approach and connected via email, which seemed to be a better way to make contact.

In hindsight, we would have offered an additional incentive for subjects to complete the 12-month followup. We also recorded self-reported weights at 12 months, which may have not been accurate.

\section{CONCLUSIONS}

This study suggests that a convenient, readily available mobile wellness coaching app with tailored individual and emotional support can be effective in improving fatigue and generating sustainable improvements in overall health and wellness habits. Wellness coaching is a novel patient-centered approach that can improve motivation and offer needed support to help breast cancer survivors achieve and sustain behavioral changes, leading to improved long-term outcomes. The results suggest that incorporation of wellness coaching as part of a breast cancer survivorship program may be useful in improving outcomes for this population.
Future randomized clinical trials are needed to explore this issue further. The long-term health care needs of breast cancer survivors are complex and further research is needed to evaluate interventions for support of their unmet needs across a wide range of domains.

\section{Patient-Friendly Recap}

- Breast cancer experts recommend that survivors maintain an optimum weight and engage in healthy lifestyle habits to reduce risk of recurrence, mortality, and other chronic diseases.

- The authors tested whether a commercially available mobile app that connects users to a personal health coach could more effectively help patients stick to healthy behaviors and their wellness plans than the standard educational approach.

- They found that patients who used the app improved their physical activity, diet, and body mass index more than the control group. Of note, at 12 months none of the app users were still using the app, which costs $\$ 65$ per month.

\section{Acknowledgments}

The Rate Your Plate tool was used with permission from Brown University Institute for Community Health Promotion (Providence, RI). Copyright 2010; all rights reserved.

\section{Author Contributions}

Study design: Cairo, Williams. Data acquisition or analysis: Cairo, Williams, Bray, Goetzke, Perez. Manuscript drafting: Cairo, Williams, Bray, Goetzke. Critical revision: Cairo, Perez.

\section{Conflicts of Interest}

None.

\section{Funding Sources}

This study received an Aurora Intramural Oncology Research Award, funded in part by the Vince Lombardi Cancer Foundation (Elm Grove, WI). The authors also acknowledge the support of the Aurora Health Care Foundation (Milwaukee, WI) and the Advocate Aurora Research Institute.

\section{References}

1. American Cancer Society. Breast Cancer Facts \& Figures, 2017-2018. American Cancer Society Inc.; 2017.

2. Cancer.Net. Breast cancer: statistics. Published February 28, 2019; accessed July 31, 2019. https://www.cancer.net/cancertypes/breast-cancer/statistics

3. Palmer SC, Blauch AN, Pucci DA, Jacobs LA. Symptom burden, unmet need for assistance, and psychosocial adaptation among longer term breast cancer survivors. (abstr.) Cancer Res. 2017;77(4 Suppl):P5-13-12. Crossref 
4. Carlson LE, Waller A, Groff SL, Giese-Davis J, Bultz BD. What goes up does not always come down: patterns of distress, physical and psychosocial morbidity in people with cancer over a one year period. Psychooncology. 2013;22:168-76. Crossref

5. Fu MR, Axelrod D, Guth AA, et al. Comorbidities and quality of life among breast cancer survivors: a prospective study. J Pers Med. 2015;5:229-42. Crossref

6. Woelfel IA, Fernandez LJ, Idowu MO, Takabe K. A high burden of comorbid conditions leads to decreased survival in breast cancer. Gland Surg. 2018;7:216-27. Crossref

7. Runowicz CD, Leach CR, Henry NL, et al. American Cancer Society/American Society of Clinical Oncology Breast Cancer Survivorship Care Guideline. CA Cancer J Clin. 2016;66:43-73. Crossref

8. McCann SE, Thompson LU, Nie J, et al. Dietary lignan intakes in relation to survival among women with breast cancer: the Western New York Exposures and Breast Cancer (WEB) Study. Breast Cancer Res Treat. 2010;122:229-35. Crossref

9. Fink BN, Weiner JG, Jordan TR, et al. Early stage breast cancer and its association with diet and exercise-related perceptions and behaviors to prevent recurrence. Breast Cancer (Auckl). 2010;4:65-72. $\underline{\text { Crossref }}$

10. Ecker BL, Lee JY, Sterner CJ, et al. Impact of obesity on breast cancer recurrence and minimal residual disease. Breast Cancer Res. 2019;21(1):41. Crossref

11. Lauby-Secretan B, Scoccianti C, Loomis D, et al. Body fatness and cancer - viewpoint of the IARC working group. $N$ Engl $J$ Med. 2016;375:794-8. Crossref

12. Vance V, Mourtzakis M, McCargar L, Hanning R. Weight gain in breast cancer survivors: prevalence, pattern and health consequences. Obes Rev. 2011;12:282-94. Crossref

13. Nichols HB, Trentham-Dietz A, Egan KM, et al. Body mass index before and after breast cancer diagnosis: associations with all-cause, breast cancer, and cardiovascular disease mortality. Cancer Epidemiol Biomarkers Prev. 2009;18:1403-9. Crossref

14. Picon-Ruiz M, Morata-Tarifa C, Valle-Goffin JJ, Friedman ER, Slingerland, JM. Obesity and adverse breast cancer risk and outcome: mechanistic insights and strategies for intervention. CA Cancer J Clin. 2017;67:378-97. Crossref

15. Ferioli M, Zauli G, Martelli AM, et al. Impact of physical exercise in cancer survivors during and after antineoplastic treatments. Oncotarget. 2018;9:14005-34. Crossref

16. Kirkham AA, Davis MK. Exercise prevention of cardiovascular disease in breast cancer survivors. J Oncol. 2015;2015:917606. Crossref

17. Spei ME, Samoli E, Bravi F, La Vecchia C, Bamia C, Benetou V. Physical activity in breast cancer survivors: a systematic review and meta-analysis on overall and breast cancer survival. Breast. 2019;44:144-52. Crossref

18. Demark-Wahnefried W, Jones LW. Promoting a healthy lifestyle among cancer survivors. Hematol Oncol Clin North Am. 2008;22:319-42. Crossref

19. Koutoukidis DA, Beeken RJ, Lopes S, Knobf MT, Lanceley A. Attitudes, challenges and needs about diet and physical activity in endometrial cancer survivors: a qualitative study. Eur J Cancer Care (Engl). 2017;26(6):e12531. Crossref
20. Bluethmann SM, Sciamanna CN, Winkels RM, Sturgeon KM, Schmitz KH. Healthy living after cancer treatment: considerations for clinical and community practice. Am J Lifestyle Med. 2018;12:215-9. Crossref

21. Terranova CO, Lawler SP, Spathonis K, Eakin EG, Reeves MM. Breast cancer survivors' experience of making weight, dietary and physical activity changes during participation in a weight loss intervention. Support Care Cancer. 2017;25:1455-63. Crossref

22. Cyarto EV, Brown WJ, Marshall AL. Retention, adherence and compliance: important considerations for home- and groupbased resistance training programs for older adults. J Sci Med Sport. 2006;9:402-12. Crossref

23. Ormel HL, van der Schoot GG, Sluiter WJ, Jalving M, Gietema JA, Walenkamp AM. Predictors of adherence to exercise interventions during and after cancer treatment: a systematic review. Psychooncology. 2018;27:713-24. Crossref

24. Simmons LA, Wolever RQ. Integrative health coaching and motivational interviewing: synergistic approaches to behavior change in healthcare. Glob Adv Health Med. 2013;2(4):28-35. Crossref

25. Barakat S, Boehmer K, Abdelrahim M, et al. Does health coaching grow capacity in cancer survivors? A systematic review. Popul Health Manag. 2018;21:63-81. Crossref

26. Duscha BD, Piner LW, Patel MP, et al. Effects of a 12-week mHealth program on peak $\mathrm{VO}_{2}$ and physical activity patterns after completing cardiac rehabilitation: a randomized controlled trial. Am Heart J. 2018;199:105-14. Crossref

27. Mao AY, Chen C, Magana C, Caballero Barajas K, Olayiwola JN. A mobile phone-based health coaching intervention for weight loss and blood pressure reduction in a national payer population: a retrospective study. JMIR Mhealth Uhealth. 2017;5(6):e80. Crossref

28. Amireault S, Godin G, Lacombe J, Sabiston CM. The use of the Godin-Shephard Leisure-Time Physical Activity Questionnaire in oncology research: a systematic review. BMC Med Res Methodol. 2015;15:60; erratum 2016;16:30. Crossref

29. Gans KM, Hixson ML, Eaton CB, Lasater TM. Rate Your Plate: a dietary assessment and educational tool for blood cholesterol control. Nutr Clin Care. 2000;3:163-9. Crossref

30. Jean-Pierre P, Figueroa-Moseley CD, Kohli S, Fiscella K, Palesh OG, Morrow GR. Assessment of cancer-related fatigue: implications for clinical diagnosis and treatment. Oncologist. 2007;12 Suppl 1:11-21. Crossref

31. Kroenke K, Spitzer RL, Williams JB. The Patient Health Questionnaire-2: validity of a two-item depression screener. Med Care. 2003;41:1284-92 Crossref

32. Bodai BI, Tuso P. Breast cancer survivorship: a comprehensive review of long-term medical issues and lifestyle recommendations. Perm J. 2015;19(2):48-79. Crossref

(C) 2020 Advocate Aurora Health, Inc. 